\title{
Diffusion of passive scalars under stochastic convection
}

\author{
A. Careta and F. Sagués \\ Departament de Química Física, Universitat de Barcelona, Diagonal 647, 08028-Barcelona, Spain
}

\begin{abstract}
J. M. Sancho
Departament d'Estructura i Constituents de la Matèria, Universitat de Barcelona, Diagonal 647, 08028-Barcelona, Spain
\end{abstract}

(Received 28 January 1993; accepted 2 September 1993)

\begin{abstract}
The diffusion of passive scalars convected by turbulent flows is addressed here. A practical procedure to obtain stochastic velocity fields with well-defined energy spectrum functions is also presented. Analytical results are derived, based on the use of stochastic differential equations, where the basic hypothesis involved refers to a rapidly decaying turbulence. These predictions are favorable compared with direct computer simulations of stochastic differential equations containing multiplicative space-time correlated noise.
\end{abstract}

\section{INTRODUCTION}

In spite of being a classical problem in statistical hydrodynamics, the diffusion of a passive scalar convected by a random incompressible fluid is still a question of major practical interest in a variety of contexts, ranging from statistical optics to diffusion controlled chemical reactions and spreading of pollutants. After the pioneering work by Taylor ${ }^{1}$ and Richardson ${ }^{2}$ in the 1920s, and Batchelor ${ }^{3}$ in the 1950s, a large body of literature ${ }^{4}$ has been devoted to the delicate question of defining and computing a turbulent diffusion coefficient as a measure of the effectiveness of turbulent mixing.

Certainly, for homogeneous flows and nonreactive scalars, one expects that such a turbulent diffusion, whenever it exits, will essentially depend on the statistical properties of the turbulence. However, there is not a unique approach to translate this expectation into analytical terms. Roberts ${ }^{5}$ applied the so-called direct interaction approximation (DIA) for one-particle diffusion, to get an expression for a turbulent diffusion coefficient, which has to be determined self-consistently using the solution of the scalar field itself. Kraichnan ${ }^{6}$ confirmed numerically the consistency of that approach using a Lagrangian description appropriate to particular prescriptions for two-dimensional (2-D) and three-dimensional Eulerian velocity fields. Analytical work and closure relations proposed by Saffman ${ }^{7}$ and McLaughlin et $a l^{8}$ has been recently generalized by Lipscombe et $a l^{9}{ }^{9}$ to account for Gaussian turbulent fields defined, not necessarily homogeneous and stationary. Finally, the more recent antecedent we are aware of reporting on this subject is that of Chechetkin et al., ${ }^{10}$ who deal with non-Gaussian fields.

The perspective we adopt here to address the problem of diffusion in random flows is quite different. ${ }^{11}$ Methodologically, the whole approach rests on the use of both analytical and simulation techniques presently available to deal with stochastic differential equations. We start with an auxiliary random scalar field $\eta(\mathbf{r}, t)$, whose temporal evolution is expressed in terms of a Langevin equation. A trivial transformation is further employed to obtain a divergence-free, isotropic, stationary, and homogeneous stochastic velocity field $\xi(r, t)$, whose statistical parameters $\left(u_{0}^{2}, t_{0}\right.$, and $1_{0}$, respectively, for its intensity, time, and length correlations), are easily identified in terms of the originally prescribed ones for $\eta(\mathbf{r}, t)$. Non-Markovian techniques, specially designed for stochastic equations with spatiotemporally correlated multiplicative noise, are finally applied to derive an evolution equation for the probability density of the dispersed scalar. The dynamics of its lowestorder moments then leads directly to the desired diffusion coefficient. The great advantage of this procedure is that the results for the effective diffusion are in this way expressed as an expansion in terms of the statistical parameters of the random velocity field.

This strategy is further checked through direct computer simulations of the previously introduced stochastic differential equations discretized on a lattice. A particular technique that largely facilitates the handling of the auxiliary field $\eta(\mathbf{r}, t)$ consists in simulating its dynamics in Fourier space, according to a procedure developed recently by some of us. ${ }^{12}$ A reasonable agreement is found between analytical and simulation results, which consistently coincide in the limit of small correlation times $t_{0}$ for slightly developed turbulent fields.

The paper is organized as follows. After these introductory remarks, Secs. II and III contain, respectively, analytical developments appropriate to the stochastic evolution for the dispersed scalar and the velocity field. In Sec. IV we summarize the results for the effective diffusion, after briefly reviewing the basic ingredients of the computer simulations. Finally, the most technical points of the analytical derivations are included in the Appendix.

\section{STOCHASTIC EQUATIONS FOR THE DISPERSED SCALAR}

Our starting point is an Eulerian equation of motion for the scalar variable $\Psi(\mathrm{r}, t)$ dispersed by a turbulent velocity field $\boldsymbol{\xi}(\mathbf{r}, t),{ }^{13}$

$$
\frac{\partial \Psi(\mathbf{r}, t)}{\partial t}=D \nabla^{2} \Psi(\mathbf{r}, t)-\nabla[\xi(\mathbf{r}, t) \Psi(\mathbf{r}, t)],
$$

$D$ is the molecular diffusion coefficient, and the velocity field is assumed to be a homogeneous, isotropic, and sta- 
tionary stochastic quantity defined by its cumulants. ${ }^{14}$ In particular, its mean value is taken zero and the second cumulant is given by (superscripts stand for vector components)

$$
\left\langle\xi^{i}\left(\mathbf{r}_{1}, t_{1}\right) \xi^{j}\left(\mathbf{r}_{2}, t_{2}\right)\right\rangle=R^{i j}(r, s),
$$

where $r=\left|\mathbf{r}_{1}-\mathbf{r}_{2}\right|$ and $s=\left|t_{1}-t_{2}\right|$. In addition, $\xi(\mathbf{r}, t)$ is assumed to represent an incompressible flow $[\nabla \xi(r, t)=0]$, so that ${ }^{15}$

$$
\frac{\partial R^{i j}(r, s)}{\partial r^{j}}=0 .
$$

Unless otherwise stated, summing over repeated indices will be employed.

The next step is to study the temporal evolution of the mean value $\langle\Psi(\mathbf{r}, t)\rangle$ averaged over the realizations of the random flow $\xi(\mathbf{r}, t)$,

$$
\frac{\partial\langle\Psi(\mathbf{r}, t)\rangle}{\partial t}=D \nabla^{2}\langle\Psi(\mathbf{r}, t)\rangle-\langle\nabla[\xi(\mathbf{r}, t) \Psi(\mathbf{r}, t)]\rangle .
$$

Actually, taking a $\delta$-like initial condition, the averaged field $\langle\Psi(r, t)\rangle$ is nothing but the probability density for the spatiotemporal distribution of a unit amount of the dispersed scalar. Its first nonzero moment,

$$
m^{i j}(t)=\left\langle r^{i} r^{j}\right\rangle_{\langle\Psi(\mathrm{r}, t)\rangle}=\int_{R^{n}} d^{n} \mathbf{r} r^{i} r^{j}\langle\Psi(\mathbf{r}, t)\rangle,
$$

is then all we need to compute an effective diffusion coefficient.

As anticipated in the Introduction, we want to compare our analytical results with those obtained from direct computer simulations of both the dynamics of the random flow and that of the dispersed scalar. Note, in passing, that this is a clear alternative to the direct simulation of the convected trajectory of a Brownian particle, an approach that was recently proposed by Drummond et al. ${ }^{16} \mathrm{Com}-$ puter simulations obviously require an appropriate discretization of the continuous scheme so far introduced. In this paper, we choose as a standard grid a 2-D square lattice of $N \times N$ points with elementary unit spacing $\Delta_{x}=\Delta_{y}=\Delta$. The updating procedure is advantageously vectorized by transforming the 2-D lattice, with positions defined by two indices $(\alpha, \beta)$, to a one-dimensional array with a single index, $\mu=(\alpha-1) N+\beta$. Shifted periodic boundary conditions are prescribed to bound the square lattice. ${ }^{17}$ Needless to say that if we also choose to perform all the analytical treatment that follows from Eqs. (1)-(5) in its discretized version, we may gain a wider range of confidence in comparing theoretical and simulation results. This is precisely what we propose on what follows, with the clear understanding that the results for the continuous theory will be easily recovered, when necessary, taking the limit $\Delta \rightarrow 0$.

Starting with this analytical program, Eq. (1) transforms into

$$
\frac{\partial \Psi_{\mu}(t)}{\partial t}=D \nabla_{\mu \nu}^{2} \Psi_{\nu}(t)+g_{\mu \nu}^{i}(t) \xi_{v}^{i}(t)
$$

with discrete centered forms for the Laplacian and partial derivative operators,

$$
\begin{aligned}
& \nabla_{\mu \nu}^{2}=\frac{1}{\Delta^{2}}\left(\delta_{\mu+1, v}+\delta_{\mu-1, v}+\delta_{\mu+N, v}+\delta_{\mu-N, v}-4 \delta_{\mu, \nu}\right), \\
& \begin{aligned}
g_{\mu \nu}^{x(y)}(t) & =-\frac{1}{2 \Delta}\left(\delta_{\mu+1(N), \hat{v}}-\delta_{\mu-1(N), \hat{v}}\right) \Psi_{\hat{\nu}}(t) \\
& =-\nabla_{\mu \hat{v}}^{x(y)} \Psi_{\hat{\nu}}(t) .
\end{aligned}
\end{aligned}
$$

In Eq. (7), notation $\hat{v}$ stands for a not contracted $v$ index. Analogously, Eq. (4) for the discretized mean value $\left\langle\Psi_{\mu}(t)\right\rangle$ reads as

$$
\frac{\partial\left\langle\Psi_{\mu}(t)\right\rangle}{\partial t}=D \nabla_{\mu \nu}^{2}\left\langle\Psi_{\nu}(t)\right\rangle+\left\langle g_{\mu \nu}^{i}(t) \xi_{\nu}^{i}(t)\right\rangle .
$$

Equation (8) is transformed by using the well-known Novikov's theorem ${ }^{18}$ into

$$
\begin{aligned}
\frac{\partial\left\langle\Psi_{\mu}(t)\right\rangle}{\partial t}= & D \nabla_{\mu \nu}^{2}\left\langle\Psi_{v}(t)\right\rangle+\int_{0}^{\infty} d t^{\prime}\left\langle\xi_{\nu}^{i}(t) \xi_{\sigma}^{j}\left(t^{\prime}\right)\right\rangle \\
& \times\left\langle\frac{\delta g_{\mu \nu}^{i}(t)}{\delta \xi_{\sigma}^{J}\left(t^{\prime}\right)}\right\rangle \\
= & D \nabla_{\mu \nu}^{2}\left\langle\Psi_{\nu}(t)\right\rangle+\int_{0}^{\infty} d t^{\prime} R^{i j}\left(r_{v, \sigma},\left|t-t^{\prime}\right|\right) \\
& \times\left\langle\frac{\partial g_{\mu \nu}^{i}(t)}{\partial \Psi_{\gamma}(t)} \frac{\delta \Psi_{\gamma}(t)}{\delta \xi_{\sigma}^{j}\left(t^{\prime}\right)}\right\rangle,
\end{aligned}
$$

where the discretized correlation tensor for the random flow $R^{i j}\left(r_{v, \sigma},\left|t-t^{\prime}\right|\right)$, and the so-called response function of the advected scalar $\delta \Psi_{\gamma}(t) / \delta \xi_{\sigma}^{j}\left(t^{\prime}\right)$ have been conveniently introduced. We recall in this point that in deriving Eq. (9), from Eq. (8) we have assumed that $\xi(\mathbf{r}, t)$ is a Gaussian stochastic field. For non-Gaussian flows one should have included higher-order cumulants. In any case, Eq. (9) must be considered as a valuable approximation for non-Gaussian velocity fields if higher-order cumulants are small enough, in comparison with $R^{i j}\left(r_{v, \sigma},\left|t-t^{\prime}\right|\right)$. It is also worth noting that in Eq. (9) we have extended the integration limits up to $\infty$, according to the fact that we are interested in dealing with a steady diffusive regime.

The main remaining task consists now in evaluating the response function left in Eq. (9). To this end one assumes that $R^{i j}\left(r_{v, \sigma},\left|t-t^{\prime}\right|\right)$ is a rapid time decaying function in units of a correlation time $t_{0}$, which is smaller than any other relevant time scale of the system. Then, an expansion of the response function in powers of $\left(t-t^{\prime}\right)$ is invoked to systematically deal with the non-Markovian dynamics expressed by Eq. (9). This development follows a general procedure valid for any prescribed noise statistics, ${ }^{19}$ and since it is of a rather technical nature we transfer it into the Appendix. Retaining only first-order contributions in $\left(t-t^{\prime}\right)$ and repeatedly summing over the lattice indices, an approximate closed equation for the probability density $\left\langle\Psi_{\mu}(t)\right\rangle$, linear in the autocorrelation 
tensor, is obtained. By referring to its spatial fluctuations $m^{i f}(t)$, a stationary diffusive regime is then identified through the usual linear law,

$$
\left\langle(\Delta r)^{2}\right\rangle=\left\langle(\Delta x)^{2}\right\rangle+\left\langle(\Delta y)^{2}\right\rangle=4(D+\Delta D) t,
$$

where $\Delta D$ is the diffusive convection originated in the turbulent flow. Explicit expressions for $\Delta D$, which can be traced back to the elementary contributions written down in the Appendix, read for the discrete version as

$$
\begin{aligned}
\Delta D_{d}= & \int_{0}^{\infty} d s R(\Delta, s)+\frac{2 D}{\Delta^{2}} \int_{0}^{\infty} d s s\left[R\left(2^{1 / 2} \Delta, s\right)\right. \\
& +R(2 \Delta, s)-2 R(\Delta, s)]
\end{aligned}
$$

where a shorthand notation has been used for the correlation tensor,

$$
R(r, s) \equiv \frac{1}{2}\left[R^{x x}(r, s)+R^{y y}(r, s)\right] .
$$

Correspondingly, the continuous version of Eq. (11) adopts the form

$$
\Delta D_{c}=\int_{0}^{\infty} d s R(0, s)+4 D \int_{0}^{\infty} d s s R^{\prime \prime}(0, s),
$$

in which $R^{\prime \prime}(0, s)=\partial^{2} R(r, s) /\left.\partial r^{2}\right|_{r=0}$. In deriving Eq. (13) from Eq. (11) we have used the symmetry properties of $R(r, s)$, which imply that $R^{\prime}(0, s)=0 .{ }^{15}$

Actually, the first contribution to $\Delta D_{c}$ in Eq. (13) is easily evaluated as $u_{0}^{2} t_{0}, u_{0}^{2}$ being any of the diagonal components of the equal-time, equal-point correlation tensor for the turbulent velocity, i.e., a measure of the turbulence intensity. Our previous treatment ${ }^{11}$ was indeed limited to that first correction, which can be also viewed as an exactly correct limiting case of Robert's analysis ${ }^{5}$ for the diffusion of a scalar field convected by a very rapidly varying random velocity field. The final output of our approach here is that the next term in the correlation time $t_{0}$ is evaluated, as expressed by Eqs. (11) and (13), written above.

To finish our analytical derivation, we only need to prescribe precise statistics for the random flow to be used in relation with the explicit results of Eqs. (11) and (13). This is exactly the aim of the next section.

\section{STATISTICS OF THE RANDOM FLOW}

As stated in Sec. II, we are interested in generating a homogeneous, isotropic, and stationary stochastic quantity representing an incompressible statistically well-behaved turbulent velocity field defined by its cumulants. In particular, the three basic parameters, $u_{0}^{2}, t_{0}$, and $l_{0}$ are easily evaluated in terms of the correlation $R(r, s)$ through their standard definitions,

$$
\begin{aligned}
& u_{0}^{2}=R(0,0), \\
& t_{0}=\frac{1}{u_{0}^{2}} \int_{0}^{\infty} d s R(0, s), \\
& l_{0}=\frac{1}{u_{0}^{2}} \int_{0}^{\infty} d r R(r, 0) .
\end{aligned}
$$

Of particular interest for us is the energy spectrum function $E(k)$ representing the density of contributions to the kinetic energy on the wave-number axis. Actually, this permits an alternative evaluation of the turbulence intensity, since

$$
u_{0}^{2}=\int_{0}^{\infty} d k E(k)
$$

The kind of spectra we are looking for is that proposed by Kraichnan, ${ }^{6}$ which describes a widely distributed band of excitations with a peak centered at a well-defined wave number $k_{0}$,

$$
E(k) \propto k^{3} \exp \left(-a k^{2} / k_{0}^{2}\right) .
$$

To this end we introduce an auxiliary random scalar field $\eta(\mathbf{r}, t)$ that satisfies the continuous Langevin equation,

$$
\tau \frac{\partial \eta(\mathbf{r}, t)}{\partial t}=-\eta(\mathbf{r}, t)+\exp \left(\frac{1}{2} \lambda^{2} \nabla^{2}\right) \xi(\mathbf{r}, t),
$$

where $\zeta(\mathbf{r}, t)$ is a Gaussian white noise of zero mean and correlation,

$$
\left\langle\zeta\left(\mathbf{r}_{1}, t_{1}\right) \zeta\left(\mathbf{r}_{2}, t_{2}\right)\right\rangle=2 \epsilon \delta\left(\mathbf{r}_{1}-\mathbf{r}_{2}\right) \delta\left(t_{1}-t_{2}\right) .
$$

Equations (17) and (18) identify the three basic statistical parameters of $\eta(\mathbf{r}, t): \epsilon$ stands for the intensity of the noise, whereas $\lambda$ and $\tau$, respectively, identify its spatial and temporal correlation lengths.

The incompressible random flow $\boldsymbol{\xi}(\mathbf{r}, t)$, in terms of which we have derived all the analytical results in the preceding section, is then simply obtained, according to

$$
\boldsymbol{\xi}(\mathbf{r}, t)=\left[\xi^{x}(\mathbf{r}, t), \xi^{y}(\mathbf{r}, t)\right]=\left(-\frac{\partial \eta(\mathbf{r}, t)}{\partial y}, \frac{\partial \eta(\mathbf{r}, t)}{\partial x}\right) .
$$

The statistics of $\boldsymbol{\xi}(\mathbf{r}, t)$ is in this way easily transformed from that of $\eta(\mathbf{r}, t)$. In particular, its Gaussian nature follows directly from that of $\zeta(\mathbf{r}, t)$, since only linear relations are involved in the definitions of both fields. The components of the random flow correlation are then expressed as

$$
\begin{aligned}
R^{i j}(r, s)= & \frac{\epsilon}{8 \pi \tau \lambda^{4}} \exp \left(-\frac{r^{2}}{4 \lambda^{2}}-\frac{s}{\tau}\right) \\
& \times\left[\frac{r^{2}}{2 \lambda^{2}} n^{i} n^{j}+\left(1-\frac{r^{2}}{2 \lambda^{2}}\right) \delta_{i j}\right],
\end{aligned}
$$

where $n^{i}$ stand for the components of the unit vector in the $\mathbf{r}_{1}-\mathbf{r}_{2}$ direction. Equation (20) verifies general symmetry properties, characteristic of homogeneous and isotropic turbulence. ${ }^{15}$ Using the above expression in Eqs. (12) and (14), we obtain, for the correlation $R(r, s)$, the result

$$
R(r, s)=u_{0}^{2} \exp \left(-\frac{\pi r^{2}}{16 l_{0}}-\frac{s}{t_{0}}\right)\left(1-\frac{\pi r^{2}}{16 l_{0}}\right),
$$

where the statistical parameters are given by 


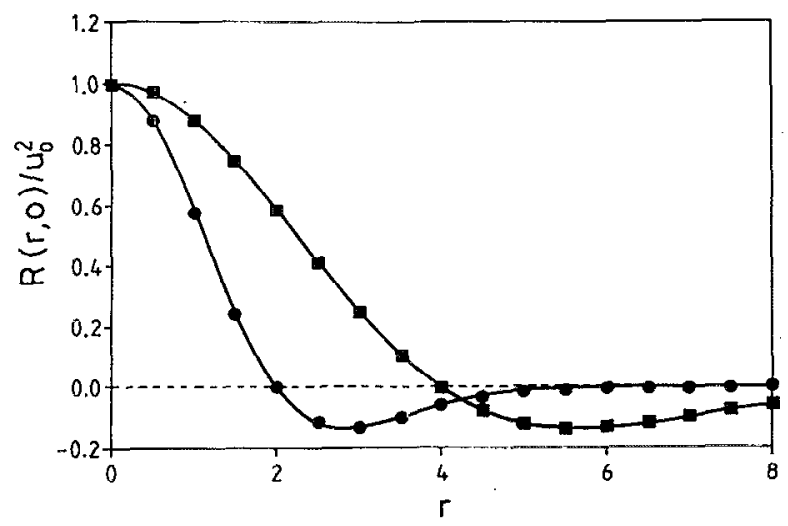

FIG. 1. Normalized correlation $R(r, 0)$ against $r$ for different values of $\lambda$. Solid lines correspond to Eq. (21) and symbols are simulation results obtained with $\epsilon=20.0, \tau=1.0, N=64, \Delta=0.5$, and $\Delta t=0.1$. The values of $\lambda$ are (-) 1.0 and (1) 2.0. The statistics are evaluated over 100 runs $\times 250$ time steps.

$$
\begin{aligned}
& u_{0}^{2}=\frac{\epsilon}{8 \pi \tau \lambda^{4}}, \\
& t_{0}=\tau \\
& l_{0}=\frac{\pi^{1 / 2}}{2} \lambda .
\end{aligned}
$$

Depicted in Fig. 1 is the behavior of $R(r, 0)$. In what refers to the energy spectrum function $E(k)$, following the prescriptions (17) and (18) given above, straightforward algebra leads directly to the result

$$
E(k)=\frac{32 u_{0}^{2} l_{0}^{4} k^{3}}{\pi^{2}} \exp \left(-\frac{4 l_{0}^{2} k^{2}}{\pi}\right),
$$

which shows a peak centered at $k_{0}=(3 \pi / 8)^{1 / 2} l_{0}^{-1}$. The characteristic shape of the energy spectrum function (23) is shown in Fig. 2.

Obviously, computer simulation of Eqs. (17) and (19) to get the stochastic flow requires their appropriate discret-

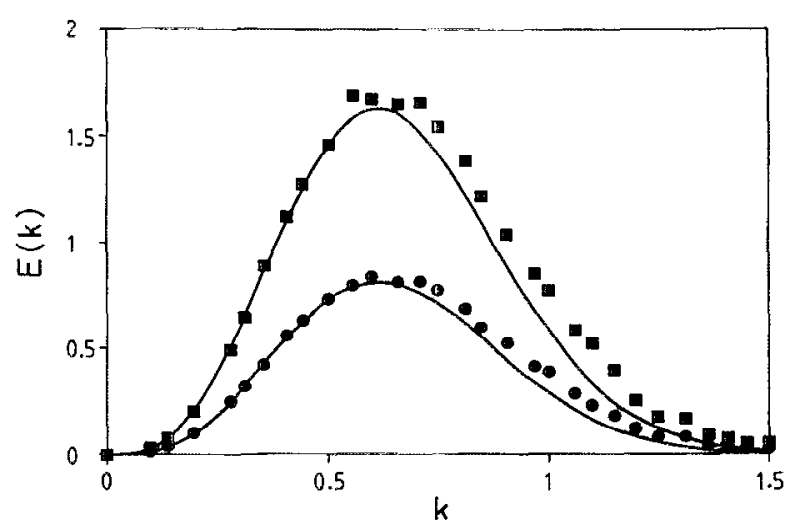

FIG. 2. Energy spectrum $E(k)$ against the wave number $k$ for different values of $\epsilon$. Solid lines correspond to Eq. (23) and symbols are simulation results obtained with $\tau=1.0, \lambda=2.0, N=64, \Delta=1.0$, and $\Delta t=0.1$. The values of $\epsilon$ are (e) 10.0 and (1) 20.0. The statistics are evaluated over 100 runs $\times 250$ time steps. ization. As anticipated in the Introduction, the simulation of the discrete auxiliary field $\eta_{\mu}(t)$ in Fourier space, largely facilitates its manipulation. The temporal evolution for the discrete wave-number components $\widetilde{\eta}_{\mu v}(t)$ derived from Eqs. (17) and (18) obeys the Langevin equation ${ }^{20}$

$$
\tau \frac{d \tilde{\eta}_{\mu \nu}(t)}{d t}=-\tilde{\eta}_{\mu \nu}(t)+\exp \left(-c_{\mu \nu}\right) \widetilde{\zeta}_{\mu \nu}(t)
$$

where

$$
c_{\mu v}=\frac{\lambda^{2}}{\Delta^{2}}\left[2-\cos \left(\frac{2 \pi \mu}{N}\right)-\cos \left(\frac{2 \pi v}{N}\right)\right],
$$

and $\widetilde{\zeta}_{\mu \nu}(t)$ is a zero mean $\delta$-anticorrelated noise, ${ }^{12}$ such that

$$
\left\langle\tilde{\xi}_{\mu v}\left(t_{1}\right) \tilde{\xi}_{\rho \sigma}\left(t_{2}\right)\right\rangle=2 \epsilon(N \Delta)^{2} \delta_{\mu,-\rho} \delta_{v,-\sigma} \delta\left(t_{1}-t_{2}\right) .
$$

For the sake of simplicity, we define in Eqs. (24)-(26) the positions in Fourier space by two indices $(\mu, v)$. Actually, the numerical running of these expressions can also be vectorized using a single index, $k=(\mu-1) N+v$. The great advantage of Eq. (24) is that it can be exactly integrated in time to give

$$
\tilde{\eta}_{\mu v}(t+\Delta t)=\tilde{\eta}_{\mu \nu}(t) \exp (-\Delta t / \tau)+\tilde{\beta}_{\mu v}(t)
$$

where $\widetilde{\beta}_{\mu v}(\ell)$ are Gaussian random numbers of zero mean and correlation,

$$
\begin{aligned}
\left\langle\widetilde{\beta}_{\mu \nu}(t) \widetilde{\beta}_{\rho \sigma}(t)\right\rangle= & \frac{\epsilon(N \Delta)^{2}}{\tau} \exp \left(-2 c_{\mu \nu}\right) \\
& \times\left[1-\exp \left(1-\frac{2 \Delta t}{\tau}\right)\right] \delta_{\mu,-\rho} \delta_{\nu,-\sigma}
\end{aligned}
$$

Once $\widetilde{\eta}_{\mu \nu}(t)$ is obtained, the appropriate transformation expressed by $\mathrm{Eq}$. (19) gives, for the discrete wave-number components of the velocity field $\widetilde{\xi}_{\mu \nu}^{i}(t)$,

$$
\begin{aligned}
& \tilde{\xi}_{\mu \nu}^{x}(t)=-\frac{i}{\Delta} \sin \left(\frac{2 \pi v}{N}\right) \tilde{\eta}_{\mu \nu}(t), \\
& \tilde{\xi}_{\mu \nu}^{y}(t)=\frac{i}{\Delta} \sin \left(\frac{2 \pi \mu}{N}\right) \tilde{\eta}_{\mu \nu}(t) .
\end{aligned}
$$

Simple algebra ${ }^{20}$ leads, for the discrete correlation,

$$
\begin{aligned}
R\left(r_{m n}, s\right)= & \frac{\epsilon}{2 \tau N^{2} \Delta^{4}} \sum_{\mu, v} \exp \left(-2 c_{\mu \nu}-\frac{s}{\tau}-i(m \mu\right. \\
& \left.+n v) \frac{2 \pi}{N}\right)\left[\sin ^{2}\left(\frac{2 \pi \mu}{N}\right)+\sin ^{2}\left(\frac{2 \pi v}{N}\right)\right],
\end{aligned}
$$

which is symmetrical when permuting $m$ and $n$, being $r_{m n}=\left(m^{2}+n^{2}\right)^{1 / 2} \Delta$. From Eq. (30) we can identify the three basic parameters of the turbulent flow. The results for $u_{0}^{2}$ and $t_{0}$ immediately read as 
$u_{0}^{2}=\frac{\epsilon}{2 \tau N^{2} \Delta^{4}} \sum_{\mu, \nu} \exp \left(-2 c_{\mu \nu}\right)\left[\sin ^{2}\left(\frac{2 \pi \mu}{N}\right)+\sin ^{2}\left(\frac{2 \pi v}{N}\right)\right]$

$t_{0}=\tau$,

whereas for $l_{0}$ we first perform a least-squares fit of $R\left(r_{m n}, 0\right)$ to the continuous result $R(r, 0)$, and then, following Eq. (14), we analytically integrate the obtained fit from zero to infinity. ${ }^{20}$ In Fig. 1 we present the obtained simulation results for $R(r, 0)$, normalized to $u_{0}^{2}$, for two different values of the correlation length $\lambda$. The continuous lines correspond to the result of Eq. (21). As can be seen, the correlation length is a convenient measure of the linear extent to which the turbulent velocities are appreciablely correlated. In what refers to the results for the energy spectrum function, Fig. 2 clearly illustrates that both the simulation and the continuous model of Eq. (23) show a welldefined peak in the wave-number space.

Now, respectively, substituting Eqs. (30) and (21) into Eqs. (11) and (13), we finally end up with systematic results for the steady effective diffusion coefficient, expressed in terms of the basic parameters $u_{0}^{2}, t_{0}$, and $l_{0}$. Explicitly they read as

$$
\begin{aligned}
\Delta D_{d}= & R(\Delta, 0) t_{0}\left[1-\frac{2 D t_{0}}{\Delta^{2}}(2\right. \\
& \left.\left.-\frac{\left[R\left(2^{1 / 2} \Delta, 0\right)+R(2 \Delta, 0)\right]}{R(\Delta, 0)}\right)+\theta\left(t_{0}^{2}\right)\right],
\end{aligned}
$$

for the discrete model or its continuous counterpart

$$
\Delta D_{c}=u_{0}^{2} t_{0}\left(1-\frac{\pi D t_{0}}{l_{0}^{2}}+\theta\left(t_{0}^{2}\right)\right)
$$

where $u_{0}^{2}$ is that of Eq. (22). Results of Eqs. (32) and (33) will be compared in Sec. IV with those resulting from computer simulations. From this comparison, it is shown that the theoretical predictions above make sense, in so much as they are consistent with globally positive corrections to the diffusion coefficient.

\section{RESULTS}

After discussing the most important steps of our analytical calculation of $\Delta D$, let us turn now to the numerical simulation scheme. Our computer simulation of Eq. (6) is runned on a square lattices of $N \times N$ points with shifted periodic boundary conditions and elementary spacing $\Delta$. The numerical procedure has two main steps: first we construct the stochastic velocity field $\xi_{\mu}(t)$ with the above prescribed properties by means of the auxiliary scalar field $\eta_{\mu}(t){ }^{20}$ The initial condition $\eta_{\mu}(0)$ is chosen to correspond to that of the steady state of $\eta_{\mu}(t)$ (understood here in a statistical sense). In this way we are sure to be in an isotropic and homogeneous velocity steady state from the beginning of the simulation. Then, we start the simulation of Eqs. (6) and (7) by means of a first-order Euler algorithm with a time-step integration $\Delta t$, which is small enough to ensure the stability of the simulation procedure,

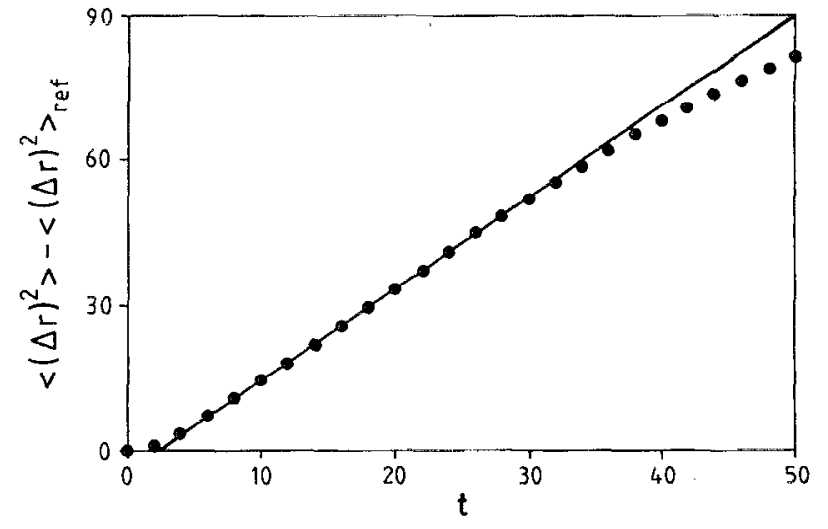

FIG. 3. Time evolution of the relative dispersion $\left\langle(\Delta r)^{2}\right\rangle-\left\langle(\Delta r)^{2}\right\rangle_{\text {ref }}$ from direct numerical simulation of Eq. (34). The straight line corresponds to the least-squares fit of Eq. (37) between $t=5$ and $t=30$, giving $\Delta D_{\text {sim }}=0.4748$. Parameter values are $D=0.1, \epsilon=15.0, \tau=0.1, \lambda=1.0$, $N=64, \Delta=0.5$, and $\Delta t=0.01$. The statistics are evaluated over 100 runs.

$$
\Psi_{\mu}(t+\Delta t)=\Psi_{\mu}(t)+\Delta t\left[D \nabla_{\mu \nu}^{2} \Psi_{\nu}(t)+g_{\mu v}^{i}(t) \xi_{v}^{i}(t)\right]
$$

with the initial condition

$$
\Psi_{\mu}(0)=\delta_{N(N-1) / 2, \mu},
$$

which represents a $\delta$-like initial condition at the center of the lattice. Now under the influence of the molecular diffusion $D$ and the velocity field, the scalar spreads over all the lattice. At each time step we measure the variance $\left\langle(\Delta r)^{2}\right\rangle$, and from its behavior in time one could directly evaluate the effective diffusion coefficient as

$$
D_{\text {eff }}=\frac{\left\langle(\Delta r)^{2}\right\rangle}{4 t} \text {. }
$$

Instead, we prefer to compute $\Delta D$ by referring to the variance in a purely diffusive system $\left\langle(\Delta r)^{2}\right\rangle_{\text {ref }}$ through a leastsquares fit to

$$
\left\langle(\Delta r)^{2}\right\rangle-\left\langle(\Delta r)^{2}\right\rangle_{\mathrm{ref}}=4 \Delta D_{\text {sim }} t,
$$

in the steady diffusive regime. In this way, the eventual nonsteady behavior of $\left\langle(\Delta r)^{2}\right\rangle$ at short and long times is better handled. Actually, some remarks are worth mentioning in relation with the temporal evolution of the variance (Fig. 3). At the beginning, the dispersed scalar needs a certain time lag to become effectively convected by the turbulent fluid. This gives rise to a transient behavior from an initial regime dominated by pure molecular diffusion to a later one, where turbulent dispersion predominates. On the other hand, as time increases, finite size effects begin to play a role, and the dispersion of the passive scalar is bounded due to the periodic boundary conditions prescribed here. As a consequence, $\Delta D$ would artificially decrease to zero. Between these two regimes, $\Delta D$ is evaluated according to the fit of Eq. (37). In Fig. 3 the least-squares fit interval ranges from $t=5-30$, including 500 simulation points.

Shown in Fig. 4 are the different results for $\Delta D$ vs $\epsilon$. Symbols correspond to numerical simulations, whereas dis- 




FIG. 4. Convective contribution $\Delta D$ to the effective diffusion coefficient against $\epsilon$. For notation and parameter values, see the text.

crete and continuous lines represent theoretical predictions, respectively, associated with Eqs. (32) and (33). In order to check the consistency of our discretization procedure with respect to the continuum limit, two different lattice configurations have been considered. Respectively, they correspond to $N=64$ with $\Delta=0.5$ (hereafter referred to as grid A) and $N=128$ with $\Delta=0.25$ (grid B). We use open symbols and dashed lines for grid A while full symbols and dotted lines stand for grid B. The two selected values of the time correlation $\tau$ are 0.1 (triangles) and 0.01 (circles). Numbering lines from bottom to top, the odd and even ones correspond respectively to $\tau=0.1$ and 0.01 . For all Fig. 4 we have fixed $D=0.1$ and $\lambda=1.0$.

In what refers to the statistics amassed, averages over 100 and 25 runs have been, respectively, used for grids $A$ and B. Both averages do not quantitatively differentiate in accuracy and are reliable enough, given the spatial average explicitly included in Eq. (5). Typical errors, estimated from individual runs, lie in the interval $0.01-0.02$ and give a maximum uncertainty for $\Delta D$ below $8 \%$.

The first significant conclusion refers to the time correlation $t_{0}$, which is directly $\tau$. As it is clearly seen, for small enough values of $t_{0}$ as those selected here, numerics entirely follow in the direction of theoretical predictions, either discrete or continuous. Actually this fact is in perfect agreement with the basic hypothesis involved in the theoretical scheme.

Second, and more important, is the question of how the continuum limit is approached. When looking at the quantitative agreement between simulation and theoretical predictions, the discrete approach always shows the best accordance with numerics, as was expected. Actually, the differences between both schemes observed in Fig. 4 are easily understood when comparing the first term on the rhs of Eqs. (32) and (33), since the correlation $R(r, s)$ at the origin $r=0$ or to first neighbors $\Delta$ could be significant different, especially for low values of $\lambda$ relative to the elementary spacing $\Delta$ (see Fig. 1). In order to diminish such differences, one is left with two possibilities: either one prescribes a smaller value for $\Delta$, while properly increasing the number of mesh points $N$, so that the continuous system has the same size, or one fixes a higher value for $\lambda$.
Unfortunately, the magnitude of $\Delta D$ for such high correlation lengths would fall under the simulation accuracy itself. Actually, the first choice corresponds to consider grid $B$ instead of grid $A$, as we have done in Fig. 4. In any case, the continuum limit is approached as the dimensionless quotient $\lambda / \Delta$ increases.

In summary, the question we have here addressed is that of the effective diffusion of passive scalars in turbulent velocity fields. In addition, a precise procedure to obtain stochastic flows with well-known energy spectrum functions is also presented. From the theoretical point of view, the basic hypothesis involved, both in a continuous and discrete model, refers to a fast correlation time of the stochastic field, allowing a controlled expansion for the effective diffusion coefficient. These predictions are favorably compared with direct computer simulations of stochastic differential equations containing multiplicative space-time correlated noise discretized on a lattice.

\section{ACKNOWLEDGMENTS}

All the calculations are performed on the IBM-3090 at CESCA (Centre de Supercomputació de Catalanya). Fruitful discussions on the numerical simulations with $J$. García-Ojalvo and L. Ramírez-Piscina are also appreciated. We acknowledge financial support from Dirección General de Investigación Científica y Técnica (Spain), under Project Nos. PB90-0030 and No. PB90-0455.

\section{APPENDIX: FORMAL EVALUATION OF RESPONSE FUNCTIONS}

The formal integration of Eq. (6) leads to

$$
\Psi_{\gamma}(t)=\Psi_{\gamma}(0)+\int_{0}^{t} d s\left[D \nabla_{\gamma \nu}^{2} \Psi_{\nu}(s)+g_{\gamma \nu}^{i}(s) \xi_{\nu}^{i}(s)\right]
$$

Deriving Eq. (A1) functionally, we get an implicit equation for the response function $\left(0<t^{\prime}<t\right)$,

$$
\begin{aligned}
\frac{\delta \Psi_{\gamma}(t)}{\delta \xi_{\sigma}^{J}\left(t^{\prime}\right)}= & g_{\gamma \sigma}^{j}\left(t^{\prime}\right)+\int_{0}^{t} d s \frac{\partial}{\partial \Psi_{l}(s)}\left[D \nabla_{\gamma \nu}^{2} \Psi_{\nu}(s)\right. \\
& \left.+g_{\gamma \nu}^{i}(s) \xi_{\nu}^{i}(s)\right] \frac{\delta \Psi_{l}(s)}{\delta \xi_{\sigma}^{j}\left(t^{\prime}\right)} .
\end{aligned}
$$

Therefore

$$
\frac{d}{d t} \frac{\delta \Psi_{\gamma}(t)}{\delta \xi_{\sigma}^{j}\left(t^{\prime}\right)}=\left.\frac{\partial}{\partial \Psi_{l}}\left(D \nabla_{\gamma \nu}^{2} \Psi_{v}+g_{\gamma \nu}^{i} \xi_{v}^{i}\right)\right|_{t} \frac{\delta \Psi_{l}(t)}{\delta \xi_{\sigma}^{j}\left(t^{\prime}\right)},
$$

which adopts the form of a differential equation, can be solved iteratively with the initial condition

$$
\left.\frac{\delta \Psi_{\gamma}(t)}{\delta \xi_{\sigma}^{j}\left(t^{\prime}\right)}\right|_{t^{\prime}=t}=g_{\gamma \sigma}^{j}(t) .
$$

The final outcome of this algebra is a series development of the response function in powers of $\left(t-t^{\prime}\right) .{ }^{19}$ Retaining only the terms independent of the turbulent field $\xi_{\mu}^{i}(t)$ up to first order in $\left(t-t^{\prime}\right)$ they read as 


$$
\frac{\delta \Psi_{\gamma}(t)}{\delta \xi_{\sigma}^{j}\left(t^{\prime}\right)}=g_{\gamma \sigma}^{j}(t)-\left(t-t^{\prime}\right) M_{\gamma \sigma}^{j}(t)+\theta\left[\left(t-t^{\prime}\right)^{2}, \xi_{\mu}^{i}(t)\right],
$$

with

$$
\begin{aligned}
M_{\gamma \sigma}^{j}(t)= & D \nabla_{\lambda \nu}^{2} \Psi_{v}(t) \frac{\partial g_{\gamma \sigma}^{j}(t)}{\partial \Psi_{\lambda}(t)} \\
& -g_{\nu \sigma}^{j}(t) \frac{\partial}{\partial \Psi_{\nu}(t)} D \nabla_{\gamma \lambda}^{2} \Psi_{\lambda}(t) .
\end{aligned}
$$

With the help of Eq. (7), Eq. (A6) transforms into

$$
M_{\gamma \sigma}^{j}(t)=-D \nabla_{\lambda \nu}^{2} \Psi_{v}(t) \nabla_{\gamma \hat{\sigma}}^{j} \delta_{\lambda \hat{\sigma}}+\nabla_{v \hat{\sigma}}^{j} \Psi_{\hat{\sigma}}(t) D \nabla_{\gamma \sigma}^{2}
$$

Now results (A5) and (A7) for the response function are directly replaced in the integral part of Eq. (9) to finally obtain the explicit evolution equation for the probability density $\left\langle\Psi_{\mu}(t)\right\rangle$. In doing so, the sort of terms that finally come from the coupled average $\left\langle g_{\mu \nu}^{i}(t) \xi_{v}^{i}(t)\right\rangle$ fall into the following two categories:

(a)

$$
\begin{aligned}
& R^{x x}\left(r_{v, \sigma^{\prime}}\left|t-t^{\prime}\right|\right)\left\langle-\nabla_{\mu \nu}^{x} \delta_{\gamma \hat{v}} g_{\gamma \sigma}^{x}(t)\right\rangle \\
& =R^{x x}\left(r_{v, \sigma^{\prime}}\left|t-t^{\prime}\right|\right)\left\langle-\nabla_{\mu \nu}^{x} \delta_{\gamma \hat{v}}\left[-\nabla_{\gamma \hat{\sigma}}^{x} \Psi_{\hat{\sigma}}(t)\right]\right\rangle \\
& =R^{x x}\left(r_{v, \sigma^{\prime}}\left|t-t^{\prime}\right|\right) \frac{1}{4 \Delta^{2}}\left\langle\left(\delta_{\mu+1, \hat{v}}-\delta_{\mu-1, \hat{v}}\right) \delta_{\gamma \hat{v}}\right. \\
& \left.\times\left(\delta_{\gamma+1, \hat{\sigma}}-\delta_{\gamma-1, \hat{\sigma}}\right) \Psi_{\hat{\sigma}}(t)\right\rangle \\
& =\frac{1}{4 \Delta^{2}}\left\langle( \delta _ { \mu + 1 , \hat { \nu } } - \delta _ { \mu - 1 , \hat { v } } ) \delta _ { \gamma \hat { v } } \left[ R^{x x}\left(r_{v, \gamma+1},\left|t-t^{\prime}\right|\right)\right.\right. \\
& \left.\left.\times \Psi_{\gamma+1}(t)-R^{x x}\left(r_{\gamma, \gamma-1},\left|t-t^{\prime}\right|\right) \Psi_{\gamma-1}(t)\right]\right\rangle \\
& =R^{x x}\left(\Delta,\left|t-t^{\prime}\right|\right) \frac{1}{4 \Delta^{2}}\left(\delta_{\mu+2, v}+\delta_{\mu-2, v}-2 \delta_{\mu, v}\right) \\
& \times\left\langle\Psi_{v}(t)\right\rangle,
\end{aligned}
$$

and similar terms for $R^{y y}$ and $R^{x y}$ (which is identical to $\left.R^{y x}\right)$. These discretized expressions reduce in the continuous limit to

$$
R^{x x}\left(0,\left|t-t^{\prime}\right|\right) \frac{\partial^{2}\langle\Psi(\mathbf{r}, t)\rangle}{\partial x^{2}}
$$

(b)

$$
\begin{aligned}
& \left(t-t^{\prime}\right) R^{x x}\left(r_{v, \sigma^{\prime}}\left|t-t^{\prime}\right|\right)\left\langle\nabla_{\mu \nu}^{x} \delta_{\gamma \hat{\nu}} M_{\gamma \sigma}^{x}(t)\right\rangle \\
& \quad=D\left(t-t^{\prime}\right) R^{x x}\left(r_{v, \sigma^{\prime}}\left|t-t^{\prime}\right|\right)\left\langle\nabla _ { \mu \hat { \nu } } ^ { x } \delta _ { \gamma \hat { \nu } } \left[-\nabla_{\lambda \nu}^{2} \Psi_{v}(t)\right.\right. \\
& \left.\left.\quad \times \nabla_{\gamma \hat{\sigma}}^{x} \delta_{\lambda \hat{\sigma}}+\nabla_{\nu \hat{\sigma}}^{x} \Psi_{\hat{\sigma}}(t) \nabla_{\gamma \sigma}^{2}\right]\right\rangle .
\end{aligned}
$$

Operating as above and avoiding to write an extremely lengthy expression for the discrete model, we get, in the continuous limit,

$$
4 D\left(t-t^{\prime}\right) R^{x x^{\prime \prime}}\left(0,\left|t-t^{\prime}\right|\right) \frac{\partial^{2}\langle\Psi(\mathbf{r}, t)\rangle}{\partial x^{2}},
$$

including the second derivative of $R^{x x}\left(r,\left|t-t^{\prime}\right|\right)$, at the origin, and similar expressions for $R^{y y}$ and $R^{x y}$.

${ }^{1}$ G. L. Taylor, "Diffusion by continuous movements," Proc. London Math. Soc. 20, 196 (1921).

${ }^{2}$ L. F. Richardson, "Atmospheric diffusion shown on a distance-neighbor graph," Proc. R. Soc. London Ser. A 110, 709 (1926).

${ }^{3}$ G. K. Batchelor, "Diffusion in a field of homogeneous turbulence. $I$. Eulerian analysis," Aust. J. Sci. Res. A 2, 437 (1949); G. K. Batchelor, "Diffusion in a field of homogeneous turbulence. II. The relative motion of particles," Proc. Cambridge Philos. Soc. 48, 345 (1952).

${ }^{4}$ For a recent review see J. C. R. Hunt, "Turbulent diffusion from sources in complex flows," Annu. Rev. Fluid. Mech. 17, 447 (1985).

${ }^{5} \mathrm{P}$. H. Roberts, "Analytical theory of turbulent diffusion," J. Fluid Mech. 11, 257 (1961).

${ }^{6} \mathrm{R}$. H. Kraichnan, "Diffusion by a random velocity field," Phys. Fluids 13, 22 (1970).

${ }^{7} \mathrm{P}$. G. Saffman, "Application of the Wiener-Hermite expansion to the diffusion of a passive scalar in a homogeneous turbulent flow," Phys. Fluids 12, 1786 (1969).

${ }^{8}$ D. W. McLaughlin, G. C. Papanicolau, and O. R. Pironneau, "Convection of microstructures and related problems," SIAM J. Appl. Math. 45, 780 (1985).

${ }^{9}$ T. C. Lipscombe, A. L. Frenkel, and D. ter Haar, "On the convection of a passive scalar by a Gaussian velocity field," J. Stat. Phys. 63, 305 (1991).

${ }^{10}$ V. R. Chechetkin, V. S. Lutovinov, and A. A. Samokhin, "On the diffusion of passive impurities in random flows," Physica A 175, 87 (1991).

${ }^{11} \mathrm{~A}$ simplified version of the approach presented here is summarized in our recent paper; A. Careta, F. Sagués, L. Ramírez-Piscina, and J. M. Sancho, "Effective diffusion in a stochastic velocity field," J. Stat. Phys. 71, 235 (1993)

${ }^{12} \mathrm{~J}$. García-Ojalvo, J. M. Sancho, and L. Ramírez-Piscina, "Generation of spatiotemporal colored noise," Phys. Rev. A 46, 4670 (1992).

${ }^{13}$ L. D. Landau and E. M. Lifshitz, Fluid Mechanics (Pergamon, Oxford, 1959).

${ }^{14}$ A. S. Monin and A. M. Yaglom, Statistical Fluid Mechanics of Turbulence (MIT Press, Cambridge, MA, 1975).

${ }^{15} \mathrm{G}$. K. Batchelor, The Theory of Homogeneous Turbulence (Cambridge U.P., Cambridge, 1970).

${ }^{16}$ I. T. Drummond, S. Duane, and R. R. Horgan, "Scalar diffusion in simulated helical turbulence with molecular diffusivity," J. Fluid Mech. 138, 75 (1984).

${ }^{17} \mathrm{~K}$. Binder and D. W. Heermann, Monte Carlo Simulation in Statistical Physics (Springer-Verlag, Derlin, 1988).

${ }^{18}$ E. A. Novikov, "Functionals and the random-force method in turbulence theory," Sov. Phys. JETP 20, 1290 (1965).

${ }^{19}$ A. Hernández-Machado, J. M. Sancho, M. San Miguel, and L. Pesquera, "Joint probability distribution of non-Markovian SDE," $\mathrm{Z}$. Phys. B 52, 335 (1983).

${ }^{20}$ An extended presentation of the simulation procedure for the stochastic velocity field will be published elsewhere. 